\title{
Receptor Chemoprint Derived Pharmacophore Model for Development of CAIX Inhibitors
}

\author{
Prakash Amresh, Kundan Kumar, Asimul Islam, Md. Imtaiyaz Hassan* and Faizan Ahmad
}

Centre for Interdisciplinary Research in Basic Sciences, Jamia Millia Islamia, Jamia Nagar, New Delhi 110025, India

\begin{abstract}
Background: Carbonic anhydrase IX (CAIX) is an attractive target for anticancer therapy because it is selectively overexpressed in tumor cells. Various CAs' inhibitors (sulfonamides/sulfaumates and coumarins) are reported as promising anti-cancer agents, showed appreciable affinity and selectivity. Novel chemical scaffolds with improved pharmacological properties are essential for the development of safe and potent CAIX inhibitors.

Materials and methods: Crystal structure of CAIX with its inhibitors revealed critical residues of CAIX that interacts to inhibitor(s). These information was used to design a receptor-chemoprint based pharmacophore model. In silico pharmacokinetic assays were carried for novel hits with both ADMET and TOPKAT tools of Discovery Studio 3.5 .

Results: Pharmacophore model consists of one hydrogen bond donor, three hydrogen bond acceptors, and two hydrophobic moieties which are defined as essential feature for CAIX inhibitors. Virtual screening of ZINC chemical databases leads to identification of 1242 hits, having pharmacophore fit score $\geq 0.95$. These hits were subsequently subjected to molecular docking analysis but they are limited to 321 only.

Conclusion: Based on consensus scoring values, critical interactions with active site residues, and predicted activity values, five compounds (ZINC03363328, ZINC08828920, ZINC12941947, ZINC03622539 and ZINC16650541) are proposed as possible lead for CAIX inhibitor. Present study suggests that a conceptual adjustment of these hits may lead to rational design of novel and potent CAIX inhibitor.
\end{abstract}

Keywords: Carbonic anhydrase IX; Anticancer therapy; Pharmacophore model; Virtual screening; CAIX inhibitors

Abbreviations: CA: Carbonic Anhydrase; ADMET: Absorption Distribution Metabolism Excretion and Toxicity; TOPKAT: Toxicity Prediction by Komputer Assisted Technology; HBD: Hydrogen Bond Donor; HBA: Hydrogen Bond Acceptor

\section{Introduction}

Carbonic anhydrase (CA) enzymes are zinc containing metalloproteins, which efficiently catalyze the reversible conversion of carbon dioxide $\left(\mathrm{CO}_{2}\right)$ to bicarbonate $\left(\mathrm{HCO}_{3}^{-}\right)$and release proton $\left(\mathrm{H}^{+}\right)$[1]. In human, all 16 different isoforms of CA belong to $\alpha$-class, and show a considerable degree of three-dimensional structural similarity with a conserved catalytic domain [2]. However, they differ widely in their cellular localization and physiological process [3]. CAs have been a potential therapeutic target for numerous pathological conditions $[1,4]$. Based on the Structure-Activity Relationship (SAR) studies, various $\alpha$-CAs' inhibitors were designed and synthesized. These inhibitors can be broadly classified into (i) inorganic anions, (ii) sulfonamides and their bioisosteres (sulfamates, sulfamides, etc.), (iii) phenols and (iv) coumarins [5-7]. These inhibitors are widely used as drug for treatment of neurological disorders, glaucoma, epilepsy, ulcer, cancer and obesity $[1,5,6]$. However, most of the currently used CA inhibitors showed cellular toxicity and scarce selectivity including various side effects $[6,8]$. Thus, it is important to develop a potential and selective CA inhibitors as a therapeutic anti-cancer agent $[4,9]$.

Among all CAs, CAIX is very peculiar, due to its limited expression in normal tissues and predominant expression in varieties of tumor cells [3]. CAIX actively participates in the regulation of $\mathrm{pH}$ in tumor cells, cell proliferation, cell adhesion, cell invasion, etc. CAIX is considered as a suitable target for cancer therapy [4,9]. Furthermore, extracellular location of this isozyme is favourable for designing selective inhibitor which can inhibit membrane associated CAs without interacting to other cytosolic and mitochondrial CAs. Recently, a high resolution crystal structure $(2.20 \AA ̊)$ of human CAIX has been determined that can be used for designing a selective inhibitor using structure based rational drug design approach $[3,9]$.

Advancements in computational techniques [8-10] played a vital role in High Throughput Screening (HTS) to design new chemical entities [11,12]. Pharmacophore modelling, Quantitative Structure Activity Relationship (QSAR), virtual screening, docking simulation and pharmacokinetics based analyses are used for development of new chemical entities [10,13]. However, the ligand based pharmacophore models are significantly depend on selected training set and conformation generation method [14]. This technique is more suitable, when protein structure or its complex with ligand has limited information [15]. Therefore, the physicochemical properties and spatial position of the active site residues of a receptor, so called "chemoprint" could be a new endeavour for the development of receptor specific potential inhibitors [16].

We have been working on designing potential ligands against

*Corresponding author: Md. Imtaiyaz Hassan, Ph. D, Centre for Interdisciplinary Research in Basic Sciences, Jamia Millia Islamia, Jamia Nagar, New Delhi 110025, India, Tel: +91-11-2698-3409; E-mail: mihassan@jmi.ac.in

Received October 03, 2013; Accepted November 17, 2013; Published November 23, 2013

Citation: Amresh P, Kumar K, Islam A, Hassan I, Ahmad F (2013) Receptor Chemoprint Derived Pharmacophore Model for Development of CAIX Inhibitors. J Carcinog Mutagen S8: 003. doi:10.4172/2157-2518.S8-003

Copyright: (C) 2013 Amresh P, et al. This is an open-access article distributed under the terms of the Creative Commons Attribution License, which permits unrestricted use, distribution, and reproduction in any medium, provided the original author and source are credited. 
various drug targets [17-20]. In the present study, docking simulations are carried out by various sets of experimentally known CAIX inhibitors, and interacting active site residues were sorted accordingly to pharmacophore features with LigandScout 3.1 [21]. Virtual screening was found to be a successful method especially when combined with molecular docking studies [10]. The predicted pharmacophore has been employed in virtual screening with a ZINC database to identify a plausible potent lead for CAIX inhibitor. The docking analysis elucidated spatial interaction of novel hit molecules. ADMET analysis is carried out for their probable pharmacokinetic profile in terms of lipophilicity $(\log \mathrm{P})$, water solubility $\left(\log \mathrm{S}_{\mathrm{w}}\right)$ and polar surface area (PSA). We have used TOPKAT tool to examine the lead molecules. Analyses of these results are quite impressive for further investigation of ZINC03363328, ZINC08828920, ZINC12941947, ZINC03622539 and ZINC16650541 as a possible lead in development of CAIX inhibitors in cancer therapy.

\section{Materials and Methods}

\section{Pharmacophore modeling}

Discovery studio 3.5 (Accelrys San Diego, USA) [22,23] and LigandScout 3.1 [21] were used to generate pharmacophore models. Based on the published literature, 25 diverse set of experimentally known CAIX inhibitors were collected from literature [1,6,7,24,25] (Figure S1) and structures were drawn with Discovery studio 3.5. Conformers of all compounds were generated using the 'best quality' option with an energy threshold of $20.0 \mathrm{kcal} \mathrm{mol}^{-1}$ and a maximum of 250 conformers sorted according to the Poling algorithm [26]. These conformational models were used in subsequent docking simulations, carried for CAIX-inhibitor complex formation according to LigandScout 3.1 manual.

LigandScout 3.1 [21] uses algorithm that allows automatic generation of 3D-pharmacophore from structural data of proteinligand complex that was used to generate 25 individual complexes based pharmacophore models. All pharmacophores were aligned together to create multi-complex-based comprehensive map to define a common and best interacting features corresponding to interacting residues and binding affinity of inhibitors with CAIX. All pharmacophore features identified by LigandScout were clustered according to their interaction pattern with the receptor. Hydrogen bond donor (HBD) and hydrogen bond acceptors (HBA) were clustered for polar interaction in terms of residues. Density-based clustering methods were used for hydrophobic, positive and negative ionizable and ring aromatic features. Amino acid residues present in vicinity of active site were clustered as excluded volume. The distance of neighboring residues from their ideal position were summed to define absolute geometric deviations. The absolute geometric deviation $\left(d_{0}\right)$ for $\mathrm{n}$ neighbors, a list of ideal points $I_{0} \ldots(n-1)$, and a list of observed points $O_{0} \ldots(n-1)$ are expressed as follows:

$$
\left.d_{a}=\sum_{i=0}^{n} \sqrt{\left(I_{i}-O_{i}\right.}\right)^{2}
$$

The relative geometric deviation $\left(d_{r}\right)$, which can be used to compare geometric deviations of different geometric bodies as shown below.

$$
\begin{aligned}
& d_{r}=\frac{d_{a}}{n} \\
& d_{a}=\frac{\sum_{i=0}^{n} \sqrt{\left(I_{i}-O_{i}\right)^{2}}}{n}
\end{aligned}
$$

Pharmacophore features showing spatial interaction with important residues of binding pocket and consistent with the crystal structure data were merged to form dynamic structure-based pharmacophore.

\section{Molecular docking}

Atomic coordinates of crystal structure of CAIX (PDB ID: 3IAI) was retrieved from Protein Data Bank (www.rcsb.org). The optimized co-ordinates of inhibitors were saved in .pdbqt format with babel-2.2.3 to carry docking analysis. All inhibitors in this study were docked into binding site of CAIX using AutoDock 4.2 [27] with standard protocol. The Lamarckian genetic algorithm (LGA) was applied to deal with protein-inhibitors interactions [28,29]. Polar hydrogen atoms were added geometrically. Kollman united atom charges were assigned to protein and PDBQT file was created. The 3-D affinity grid fields with grid map of $60 \times 80 \times 60$ points were created using the auxiliary program AutoGrid. A grid-point spacing of $0.375 \AA$ (roughly a quarter of the length of a carbon-carbon single bond) and a distance-dependent function of the dielectric constant was used for the calculation of energetic map. Other parameters were defined accordingly to our previous report [30,31]. PASS method with Perl script was applied to calculate the center of mass (COM) of the active site [32]. For evaluating binding energy in the docking step, Columbic electrostatic potential, van der Waals interaction represented as a Lennard-Jones12-6 dispersion/repulsion term and hydrogen bonding represented as a directional 12-10 term were taken into account. The most favorable free energy of binding were attained by considering docking orientations lying within the range of $2.0 \AA$ in root-mean square deviation (rmsd) tolerance and clustering each other to get result. Top posed docking conformations obtained were subjected to post-docking energy minimization on Discovery Studio 3.5. All calculations were carried out on workstation machines running Linux x86 as operating systems. The resultant structure files were analyzed using PyMOL visualization programs [33]. The receptor-inhibitors complexes were used to define the pharmacophore model.

\section{Database search and hits selection}

Best aligned CAIX-pharmacophore model was used as a 3-D query for searching potent compounds from ZINC chemical database having approximately $2 \times 10^{8}$ compounds. Database screening was performed using ligand pharmacophore mapping protocol [34]. Compounds which fitted minimum ( $\mathrm{n}-2 ; \mathrm{n}$ is total feature of present pharmacophore) pharmacophore features were considered as hits. Docking simulation was carried to validate and reduce hit molecules. These molecules were further filtered with ADMET (Absorption, Distribution, Metabolism, Excretion, and Toxicity properties) and TOPKAT (TOxicity Prediction by Komputer Assisted Technology) tools of DS3.5 [22,23].

\section{Results and Discussions}

\section{Docking simulation}

The docking simulations were carried out with CAIX inhibitors to identify the active site residues involved in inhibitors interaction to define a chemoprint used for comprehensive mapping of pharmacophore (Supplementary Table S1). Docking results showed that CAIX active site is located in a large conical cavity which spans from the surface to the center of the protein and inhibitors spatially fits horizontally (12-14 $\AA$ ). The zinc ion $\left(\mathrm{Zn}^{+2}\right)$ is located at the bottom of this cavity surrounded by His-64, His-94 and His-119. The two distinct hydrophobic ends with core having hydrophilic amino acids restrict the active site of CAIX. The lower hydrophobic domain is defined with Leu-91, Leu-93, Leu-198, Val-121, Leu135, while upper hydrophobic 
Citation: Amresh P, Kumar K, Islam A, Hassan I, Ahmad F (2013) Receptor Chemoprint Derived Pharmacophore Model for Development of CAIX Inhibitors. J Carcinog Mutagen S8: 003. doi:10.4172/2157-2518.S8-003

region consists of hydrophobic amino acid residues (Leu-141, Val-143, Pro-201, Pro-202). Trp-5, Tyr-7, Trp-209 and Phe-245 are involved in ring aromatic, $\pi-\pi$ interaction (Figure $1 \mathrm{~A}$ ). In the crystal structure, Leu-91, Val-121, Val-131, Leu-135, Leu-141, Val-143, Leu-198, and
Pro-202 define a hydrophobic region, while Asn-62, His-64, Ser-65, Gln-67, Thr-69, and Gln-92 have been identified as hydrophilic active site residues [3]. However, docking orientation of CAIX inhibitors also showed many polar interactions with His-94, His-119, Glu-106,
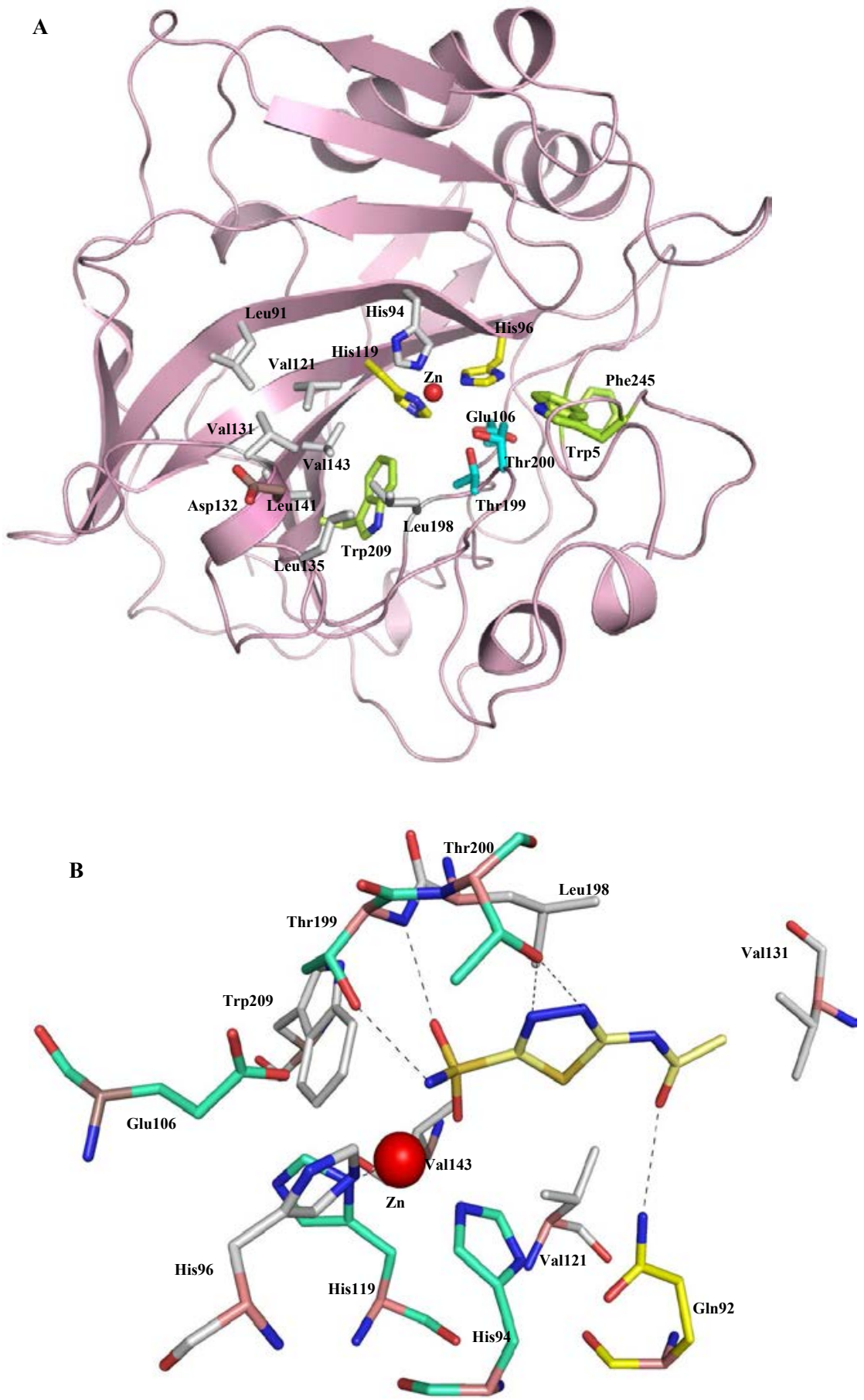

Figure 1: (A) Overall structure of CAIX is shown in cartoon model. The active site residues interacts with inhibitors are shown in sticks. The lower hydrophobic domain of active site is describe with Leu-91, Leu-93, Leu-198, Val-121, Leu135, while upper hydrophobic region constituted with Leu-141, Val-143, Pro-201, Pro-202. The amino acid residues, Trp-5, Trp-209 and Phe-245 are involved in ring aromatic interaction. His-96 and His-119 are defined as HBD. HBA feature was described as Glu-106, Thr-199 and Thr-200, His-94 and His-96. The centrally coordinated zinc atom $\left(\mathrm{Zn}^{+2}\right)$ is shown in red sphere, may acts as positive ionisable/HBD feature. The negative ionisable feature is represented by Asp-132. Interacting features of active site residues are exported to LigandScout 3.1 was used for generation of CAIX inhibitor pharmacophore model. Structure was drawn in PyMol using atomic coordinates of PDB 3IAI [6]. (B) Docked pose of CAIX inhibitor acetazolamide (AAZ), represented in stick model. The active site residues, Gln-92, His-94, His-119, Thr-199 and Thr-200 are involved in polar interactions are illustrated in stick model. Leu198, Val-121, 131, 143 and Trp-209 are involved in hydrophobic and aromatic interactions, respectively. Polar interaction showed in black dotted line. 
Thr-199 and Thr-200. In these His-94 and His-119 were defined as HBD, and Glu-106, Thr-199 and Thr-200 were designated as HBA. Docked poses of CAIX inhibitor acetazolamide (AAZ) resembled spatial orientation as in crystal structure (PDB ID: 3IAI) (Figure 1B) and showed a reasonable rmsd value of 0.23 . Correlation coefficient graph of binding affinity of CAIX inhibitors is shown in Figure 2. A high level of correlation $\left(r^{2}=0.92\right)$ is observed between the experimental and predicted binding affinity of CAIX inhibitors. The docking score and pharmacophoric feature of all selected inhibitors are given in Supplementary Table S2. It was observed that docked scores are very close to the experiments value [1,3,4]. Residues involved in ligand interactions were sorted according to pharmacophoric feature. The best-scored docking pose of all CAIX inhibitors were imported to LigandScout for the generation of pharmacophore.

\section{Structure based pharmacophore modeling}

Resulting CAIX inhibitor(s) complex was (were) used for identification of pharmacophore features. These features were obtained as a collection of all possible combinations of six features: HBD, PI (positive ionisable), HBA, $\mathrm{H}$ (hydrophobic), AR (aromatic ring) and NI (negative ionisable) in three-dimensions. The best quantitative pharmacophore model showed HBA (3), HBD (2), PI (1), H (2) and AR (1) as essential 3-D features of potent CAIX inhibitors (Figure 3A). AAZ mapped well to CAIX pharmacophore with pharmacophore fit score 0.91 (Figure $3 \mathrm{~B})$. The methy $\left(-\mathrm{CH}_{3}\right)$ of acetamide preferentially mapped with hydrophobic feature, oxygen and amino of acetamide mapped with HBA and HBD. Thiadiazol ring fitted with AR and sulfonamide moiety mapped with $\mathrm{HBD}$ and HBA features of pharmacophore. The pharmacophore mapped 2-D feature description of AAZ is shown in Figure 3D. Thiadiazol ring 3, 4 nitrogens $(\mathrm{N})$, both are accurately fitted with HBA and amino $\left(-\mathrm{NH}_{2}\right)$ of acetamide as well as sulfonamide exhibited HBD feature. For a full pharmacophore map, it is also important to include excluded volume features which reflect potential steric restriction and correspond to the positions that are inaccessible to ligands. Among four selected residues one hydrogen bond donor (Asn-62) and three hydrogen bond acceptor (Gln-67, Glu106 and Thr-199) are defined as pharmacophore features.

The LigandScout approach allows the fast generation of active site residues based pharmacophore models for development of CAIX inhibitors. The method captures CAs inhibitors spatial fitting in the

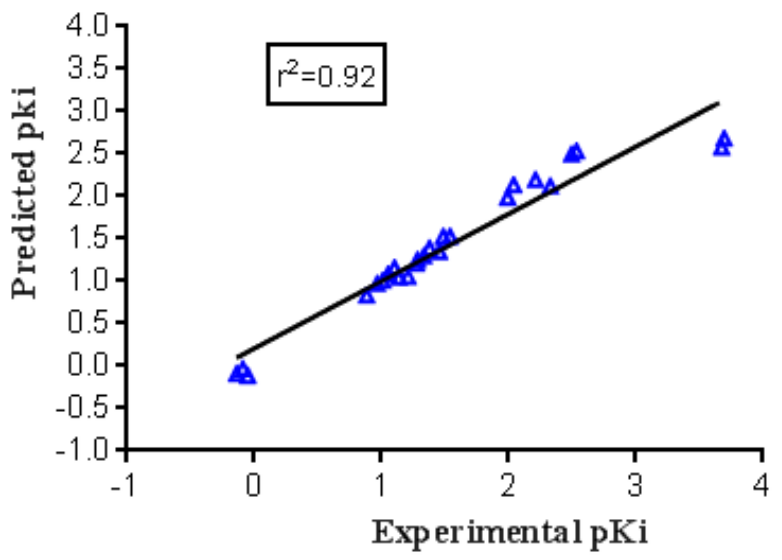

Figure 2: The correlation diagrams between experimental and predicted binding affinity $(\mathrm{nM})$ of CAIX inhibitors. $P$ value $<0.0001$ and Correlation coefficient $\left(r^{2}\right)=0.92$ active site and their interaction with residues as pharmacophore feature. Recently, ligand based CAIX pharmacophore model has suggested that HBA-(3) and HBD-(1) are essential features for CAIX inhibitor, which is consistent with the present pharmacophore. However, other essential features like PI, AR and $\mathrm{H}$ of our model were completely missing there [35]. Moreover, active site of CAIX is characterized by two distinct hydrophobic domains (extended $\approx 12 \AA$ ) with core electron rich region involving various polar interactions, which is well described in the present model. Results suggest that hydrophobic feature may act as a decisive component to enhance the activity and selectivity of CAIX inhibitors. Furthermore, orientation and localization of CAIX active site residues (Gln-67, Thr-69, Leu-91, Val-131, Asp-132, Leu-135, Gly136, and Ala-204) that differed significantly among other CAs, $[2,3]$ are well described in our model (Figures 1 and 3). Residues, Gln-67 and Thr-69 defined as HBA, and Asp-132, is designated with PI feature. Leu-91, Val-131, Asp-132, Leu-135 are define as hydrophobic feature. Physiochemical properties of the active site residues and selected pharmacophore features are consistent with CAIX crystal structure $[4,6,8]$ (Figure $1 \mathrm{~A}$ and Supplementary Table S1).

This pharmacophore is a true representative of CAIX active site, and chemoprint based method showed superior efficiency than earlier ligand based pharmacophore model $[8,35]$, where quality of model affected significantly with training data set selection and conformation generation method [14,15]. Moreover, Catalyst tool includes ligand information only to define the pharmacophoric feature constraint, whereas in LigandScout, the spatial complementarities of ligands with physiochemical properties of active site residues are used to determine the pharmacophore features [36]. All selected novel hits are well spatially fitted with pharmacophore and showed relative pharmacophore fit hit $\geq 0.95$.

\section{Virtual screening}

Best fitting pharmacophore model was used as a 3-D structural query for retrieving potential CAIX inhibitors from ZINC chemical databases, which consists of $2 \times 10^{8}$ compounds. As a result, a total of 1242 compounds which showed good mapping with pharmacophore (relative pharmacophore fit score $>0.95$ ), are selected and subsequently subjected to molecular docking analysis. A total of 321 hits showed an appreciable binding affinity with CAIX. The top ranked 10 molecules, have binding affinity $(\Delta \mathrm{G} \leq 10 \mathrm{kcal} / \mathrm{mol})$ and estimated inhibition constant $(\mathrm{Ki} \leq 10 \mathrm{nM})$ are shown in Supplementary Table S2. The best 10 screened compounds (Figure 4) with their pharmacophore fit scores are given in Supplementary Table S3). All novel hits are accurately fitted within CAIX active site and their interactions were comparable to those of AAZ [3]. The predicted hits are further evaluated with ADMET and TOPKAT tools of Discovery Studio 3.5.

\section{Pharmacokinetics and toxicity}

Most of drug molecules fail in clinical trials due to weak pharmacokinetic profile and cellular toxicity. Therefore, in silico pharmacokinetic profile of selected hits was evaluated to address the putative bioavailability to CAIX inhibitors (Table 1). Physicochemical properties, especially aqueous solubility $(\log S)$, lipophilicity $(\log \mathrm{P})$, polar surface area (PSA), and molecular weight (MW) are directly associated with absorption and bioavailability of a drug molecule [13]. These properties directly affects the movement of a drug from the site of administration into the blood. Other descriptor CYPs (cytochrome P450), an important family of biotransformation enzymes, play a crucial role in drug metabolism and are equally important for disposition of drugs, their pharmacological and 
Citation: Amresh P, Kumar K, Islam A, Hassan I, Ahmad F (2013) Receptor Chemoprint Derived Pharmacophore Model for Development of CAIX Inhibitors. J Carcinog Mutagen S8: 003. doi:10.4172/2157-2518.S8-003

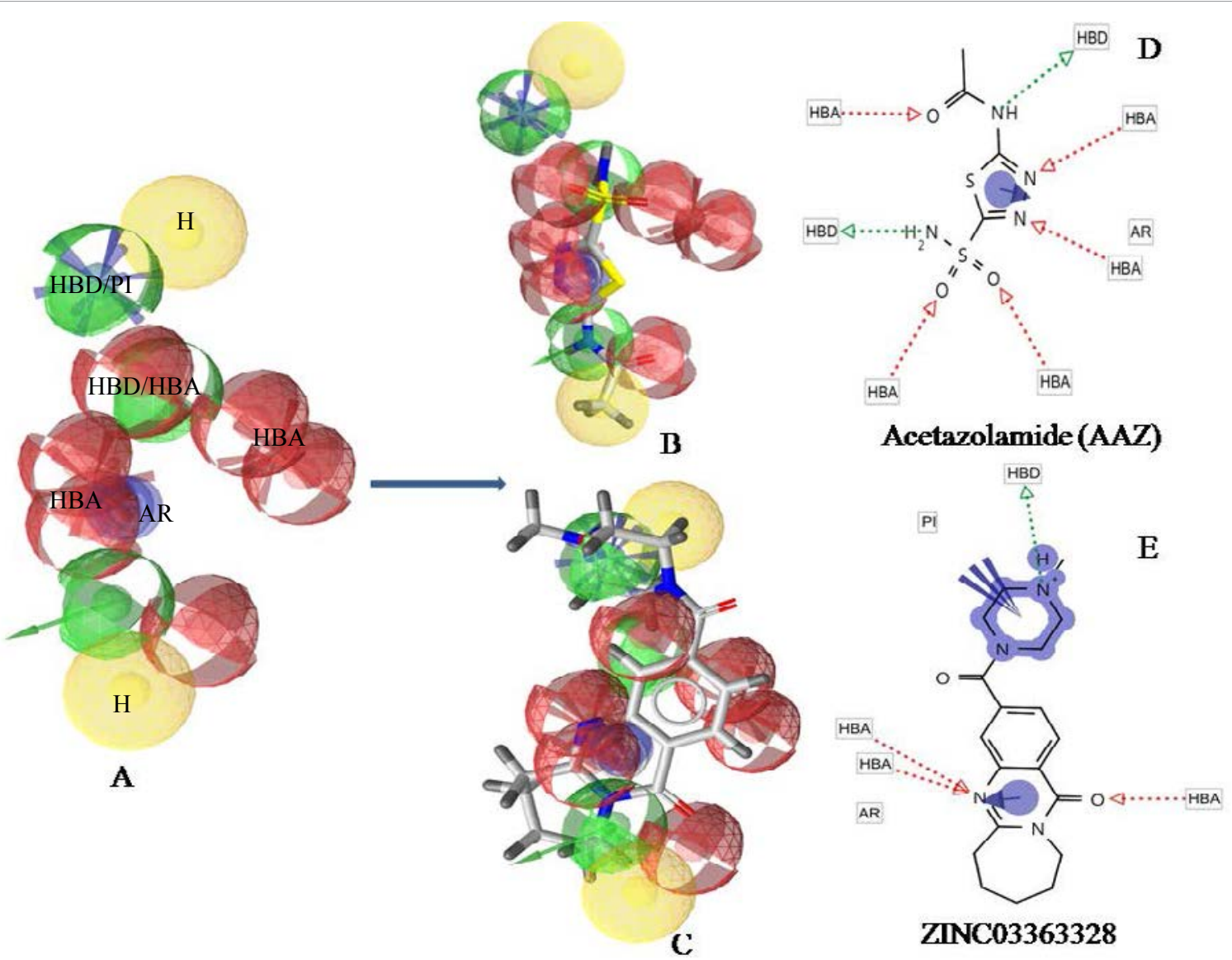

Figure 3: (A) Pharmacophore model for CAIX inhibitor. HBD (hydrogen bond donor) is shown in green, PI (positive ionisable) shown in blue, HBA (hydrogen bond acceptor) shown in red, $\mathrm{H}$ (hydrophobic) in yellow and $\mathrm{AR}$ (aromatic ring) is shown in blue ring. $\mathrm{HBD} / \mathrm{PI}$ and $\mathrm{HBD} / \mathrm{HBA}$ represent that both features are aligned at same loci and equally important. (B) CAIX inhibitor acetazolamide (AAZ) mapped with pharmacophore model. The methyl (-CH . $_{3}$ of acetamide preferentially mapped with hydrophobic feature, oxygen and amino of acetamide mapped with HBA and HBD. Thiadiazol ring fitted with AR. Sulfonamide moiety mapped with HBD and HBA feature of pharmacophore (C) The best novel hit ZINC03363328 mapped with CAIX inhibitor pharmacophore. Methylpiperazine fitted PI and HBD feature, quinazolin ring fix with $A R, H B A$ whereas azepane ring and methyl of piperazine ring mapped with hydrophobic feature. (D and $E)$ CAIX-Pharmacophore mapped 2D feature description of AAZ and ZINC03363328 at LigandScout 3.1.<smiles>CN1CCN(C(=O)c2ccc3c(=O)n4c(nc3c2)CCCCC4)CC1</smiles>

1. ZINC03363328<smiles>CCC(=O)Nc1nc(=S)ss1</smiles>

2. ZINC08828920<smiles>CC(=O)c1ccc2c(c1N)OCCO2</smiles>

3. ZINC03622539<smiles>COc1c(O)ccc(C(C)=O)c1N</smiles>

4. ZINC00494463<smiles>COc1ccc(C(=O)N2CCCCCC2)c(O)c1</smiles>

5. ZINC12941947<smiles>CC(=O)C1=C(C)OC(N)=C(C#N)C1c1ccc(O)cc1</smiles><smiles>CC(=O)Nc1ccc(C(=O)C(C)Cl)cc1[N+](=O)[O-]</smiles>

7. ZINC32118378

6. ZINC00284720<smiles></smiles><smiles>CCCC(C)NC(=O)c1ccc(NC(=O)c2ccco2)cc1</smiles>

9. ZINC16601509

10. ZINC16650541

Figure 4: The 2-D structure of best 10 novel hits. 


\begin{tabular}{|c|c|c|c|c|c|c|c|c|c|c|c|c|c|c|}
\hline \multicolumn{11}{|c|}{ ADMET } & \multicolumn{4}{|c|}{ TOPKAT } \\
\hline S. No. & Compounds & BBB & $A \log P$ & Sol. & HIA & HTL & HT_Prob & PPB & CYP2D6 & PSA & Ames Mut. & Prob. & Enrichment & WOE \\
\hline \multicolumn{15}{|c|}{ Novel hit molecules } \\
\hline 01 & ZINC03363328 & 3 & 0.013 & 3 & 0 & 0 & 0.05 & 0 & 0 & 55.83 & NM & 0.38 & 0.69 & NC \\
\hline 02 & ZINC08828920 & 1 & 2.966 & 3 & 0 & 0 & 0.29 & 0 & 0 & 41.43 & NM & 0.66 & 1.18 & NC \\
\hline 03 & ZINC03622539 & 3 & 0.609 & 4 & 0 & 0 & 0.17 & 0 & 0 & 61.70 & NM & 0.54 & 0.97 & NC \\
\hline 04 & ZINC00494463 & 2 & 1.978 & 3 & 0 & 0 & 0.42 & 2 & 0 & 47.05 & NM & 0.60 & 1.07 & C \\
\hline 05 & ZINC12941947 & 2 & 2.358 & 3 & 0 & 0 & 0.14 & 0 & 0 & 50.39 & NM & 0.46 & 0.82 & NC \\
\hline 06 & ZINC00284720 & 3 & 1.247 & 3 & 0 & 1 & 0.92 & 2 & 1 & 96.52 & NM & 0.32 & 0.57 & NC \\
\hline 07 & ZINC32118378 & 3 & 0.298 & 4 & 0 & 1 & 0.52 & 0 & 0 & 93.74 & M & 0.76 & 1.37 & C \\
\hline 08 & ZINC14183954 & 2 & 3.69 & 2 & 0 & 0 & 0.43 & 2 & 0 & 65.70 & M & 0.77 & 1.39 & NC \\
\hline 09 & ZINC16601509 & 2 & 2.92 & 3 & 0 & 1 & 0.77 & 0 & 1 & 72.77 & NM & 0.60 & 1.08 & C \\
\hline 10 & ZINC16650541 & 4 & 1.55 & 4 & 1 & 0 & 0.13 & 2 & 0 & 83.67 & NM & 0.67 & 1.21 & NC \\
\hline \multicolumn{15}{|c|}{ CAIX Inhibitors } \\
\hline 01 & Acetazolamide & 4 & 0.088 & 3 & 0 & 1 & 0.582 & 0 & 0 & 113.77 & NM & 0.43 & 0.78 & NC \\
\hline 02 & Methazolamide & 3 & 0.411 & 3 & 0 & 1 & 0.602 & 0 & 0 & 84.95 & NM & 0.72 & 1.29 & C \\
\hline 03 & Ethoxzolamide & 3 & 0.355 & 3 & 0 & 0 & 0.251 & 0 & 0 & 103.53 & NM & 0.21 & 0.38 & NC \\
\hline 04 & Sulthiame & 3 & 1.083 & 3 & 0 & 1 & 0.609 & 1 & 0 & 96.30 & NM & 0.16 & 0.28 & C \\
\hline 05 & Dichlorophenamide & 3 & 2.918 & 2 & 0 & 0 & 0.205 & 2 & 0 & 87.37 & NM & 4.37 & 7.83 & C \\
\hline 06 & Dorzolamide & 2 & 4.428 & 1 & 0 & 1 & 0.854 & 1 & 0 & 77.75 & NM & 0.37 & 0.66 & NC \\
\hline 07 & Brinzolamide & 3 & 2.6 & 2 & 0 & 1 & 0.748 & 1 & 1 & 84.95 & NM & 0.45 & 0.82 & NC \\
\hline 08 & Indisulam & 3 & 0.359 & 4 & 0 & 1 & 0.503 & 1 & 0 & 64.71 & NM & 0.19 & 0.34 & NC \\
\hline 09 & Topiramate & 1 & 1.899 & 3 & 0 & 1 & 0.735 & 2 & 0 & 26.23 & NM & 0.62 & 1.12 & C \\
\hline 10 & Zonisamide & 2 & 4.22 & 2 & 0 & 1 & 0.609 & 1 & 0 & 83.40 & NM & 0.59 & 1.07 & NC \\
\hline 11 & Sulpiride & 4 & 5.084 & 1 & 1 & 1 & 0.966 & 2 & 0 & 93.31 & NM & 0.61 & 1.09 & NC \\
\hline 12 & Coumate & 3 & 0.03 & 4 & 0 & 1 & 0.695 & 0 & 0 & 104.44 & NM & 0.20 & 0.37 & NC \\
\hline 13 & Emate & 2 & 2.904 & 2 & 0 & 0 & 0.437 & 0 & 0 & 72.73 & NM & 0.07 & 0.12 & C \\
\hline 14 & Celecoxib & 2 & 3.234 & 2 & 0 & 1 & 0.708 & 0 & 0 & 81.66 & NM & 0.52 & 0.94 & C \\
\hline 15 & Valdecoxib & 2 & 3.442 & 2 & 0 & 0 & 0.45 & 0 & 0 & 63.80 & NM & 0.64 & 1.16 & C \\
\hline 16 & Saccharin & 2 & 2.946 & 3 & 0 & 1 & 0.801 & 2 & 0 & 72.73 & NM & 0.48 & 0.86 & C \\
\hline 17 & Coumarins & 4 & -0.748 & 3 & 3 & 1 & 0.682 & 0 & 0 & 157.30 & NM & 0.54 & 0.97 & $\mathrm{NC}$ \\
\hline 18 & Imatinib & 4 & 1.386 & 3 & 0 & 0 & 0.443 & 0 & 0 & 114.34 & NM & 0.53 & 0.96 & NC \\
\hline 19 & Nilotinib & 0 & 3.816 & 2 & 0 & 1 & 0.834 & 1 & 0 & 0 & M & 0.76 & 1.37 & C \\
\hline 20 & Omeprazole & 3 & 1.823 & 3 & 0 & 1 & 0.556 & 0 & 0 & 81.33 & NM & 0.69 & 1.24 & NC \\
\hline 21 & Pantoprazole & 3 & 0.113 & 3 & 0 & 0 & 0.271 & 0 & 0 & 99.09 & NM & 0.45 & 0.82 & NC \\
\hline 22 & Lansoprazole & 4 & 0.569 & 3 & 0 & 1 & 0.582 & 0 & 0 & 122.28 & NM & 0.38 & 0.68 & NC \\
\hline 23 & Rabeprazole & 3 & 1.134 & 3 & 0 & 0 & 0.264 & 0 & 0 & 108.55 & NM & 0.58 & 1.05 & $\mathrm{NC}$ \\
\hline 24 & Amiloride & 4 & -0.307 & 3 & 0 & 1 & 0.503 & 0 & 0 & 120.83 & NM & 0.36 & 0.64 & NC \\
\hline 25 & Cariporide & 4 & 1.98 & 2 & 0 & 1 & 0.721 & 2 & 0 & 123.60 & NM & 0.28 & 0.51 & NC \\
\hline
\end{tabular}

BBB: Blood Brain Barrier Level; Sol.: Solubility Level; HIA: Human Intestinal Absorption Level; HTL: Hepatotoxicity Level; HepTox Prob.: Hepatotoxicity Probability; Prob.: Probability; Mut: Ames mutagen prediction; Prob.: Ames probability; Enrichment (Ames enrichment); WOE-_Prediction (weight of evidence); M: (Mutagen); NM: (NonMutagen); C: (Carcinogen); NC: (Non-Carcinogen). The BBB (blood brain barrier) level 0-4, having high penetration to no penetration, absorption level ideal value range from $0-1$ as good to moderate, Ideal value of solubility level is 3 , Hepatotoxicity probability<0.5 is ideal, similarly probability value for CYP2D6<0.5 is good and denoted with level 0 , plasma protein binding value 0 is good and a compounds to accessible with BBB, AlogP value should not be greater than 3.0 and polar surface area $\leq 100$ is ideal.

\section{Table 1: Pharmacokinetics profile of CAIX inhibitors and novel hits}

toxicological effects [37]. Here, ADMET (DS3.5) tool was employed to compute the probable pharmacokinetic profile of molecules. It uses QSAR models to estimate a range of ADMET related properties for small molecules. Results showed that all virtual hit molecules have ideal AlogP value $\leq 3$, except ZINC14183954 ( $\log \mathrm{P}=3.69)$. However, $\log \mathrm{P}$ value upto 4 is considerable for a drug molecule. Similarly, all hits showed good to moderate range of solubility level (solubility level=3 to 4). The best novel hit (ZINC03363328) showed better solubility with level 3, $A \log \mathrm{P}=0.013$, having good absorption level 0 and minimum hepatotoxicity probability, i.e., 0.05 . Only three compounds (ZINC00284720, 32118378, 16601509) showed hepatotoxicity probability score $\geq 0.5$. The observed human intestinal absorption (HIA) value is good for all molecules, except ZINC16650541 having HIA level=1. The blood-brain-barrier (BBB) penetration ability of compounds are categorized in four prediction levels $0-4$, having high penetration to no penetration respectively. All hit molecules showed better ability, except ZINC16650541 (BBB=4). The known CA inhibitors, AAZ, methazolamide (MZA), ethoxzolamide (EZA), dichlorophenamide (DCP) and dorzolamide (DZA), all are in clinical application showed good $\log \mathrm{P}$ value $\leq 3$, except DZA. All have good human intestinal absorption value, however, AAZ, MZA and DZA showed higher probability score of hepatotoxicity. The antiglaucoma drug brinzolamide (BRZ) having a good ADMET profile $(\log \mathrm{P}=2.6$, $\mathrm{BBB}=3$, solubility $=2$ and $\mathrm{HIA}=0$ ), showed hypotoxicity effect and is also reactive to CYP2D6. The CYP2D6 probability of all hits showed $<0.5$, except ZINC00284720 and ZINC16601509, demonstrated that all compounds were non-inhibitor to CYP2D6 enzyme. For good druggability, the ideal PPB (plasma protein binding) level is 0 . All hit compounds, except 4, 6, 8, and 10, showed better PPB activity and come under level-0, whereas DCP, DZA and BRZ showed higher score values (level-1 and 2). PSA is dependent on the conformation and possible internal hydrogen-bonding. It implies the single low-energy 
Citation: Amresh P, Kumar K, Islam A, Hassan I, Ahmad F (2013) Receptor Chemoprint Derived Pharmacophore Model for Development of CAIX Inhibitors. J Carcinog Mutagen S8: 003. doi:10.4172/2157-2518.S8-003

conformer of the molecule. For drug activity, the optimum value of PSA $\leq 90 \AA^{2}$ is well defined [38]. The hydrogen bonding and $\log \mathrm{P}$ are the main two descriptors to define the PSA of molecule. All predicted compounds showed appreciable PSA, whereas AAZ and EZA showed higher PSA value $\geq 90 \AA^{2}$.

The computer-aided toxicity predictor, TOPKAT was used to predict the cellular toxicity of novel hits. Our primary emphasis was to compute the mutagen and carcinogenic effect of molecules with Ames Prediction and WOE Prediction (weight of evidence). However, it includes varieties of models and toxicity endpoints (teratogenicity, irritation, sensitization, immunotoxicology and neuro-toxicity) that are often used in drug development. All selected molecules showed Ames probability score $\leq 7$ and are non-mutagen except ZINC32118378 and ZINC14183954 having probability score $\geq 7$ and are anticipated as mutagen. All CA inhibitors under study are predicted as non-mutagen, except nilotinib. Other toxicity predictor WOE (weight of evidence) was used to determine the relative level of certainty of compounds/agents that may cause cancer in human. All compounds were found as noncarcinogenic, except three virtual hits ZINC00494463, ZINC32118378 and ZINC16601509. MZA and DCP are predicted as carcinogenic. The comparative ADMET score and TOPKAT property data of virtual hits with standard drug suggested that selected molecule may be exploited as bioactive molecules.

\section{Molecular properties of lead hits}

HTS of ZINC database and their docking analysis led to the identification of 10 novel hits for CAIX inhibitor. In spite of having a good ADMET score, two compounds showed mutagen effect and three are anticipated as a carcinogen in TOPKAT test (Table 1). Among 10 hits, only five hits (ZINC03363328, ZINC08828920, ZINC12941947, ZINC03622539 and ZINC16650541) showing better ADMET and TOPKAT values, are considered as possible lead molecules (Figure 4). All novel hit compounds preferably fitted with CAIX pharmacophore with fit score $\geq 0.95$. The best novel hit ZINC03363328 with fit score 0.97 observed here (Figures 3C and 3E), showed most favourable score for all computational analyses. It is because of the presence of quinazoline derivative having piperazine scaffold at one end and azepane ring at the other end. The quinazoline is one of the most widespread chemical scaffolds amongst bioactive compounds and shown a promising results in the development of novel anti-cancer drug molecules [39]. Whereas azepane derivatives possesses anti-cell-proliferation activity [40].

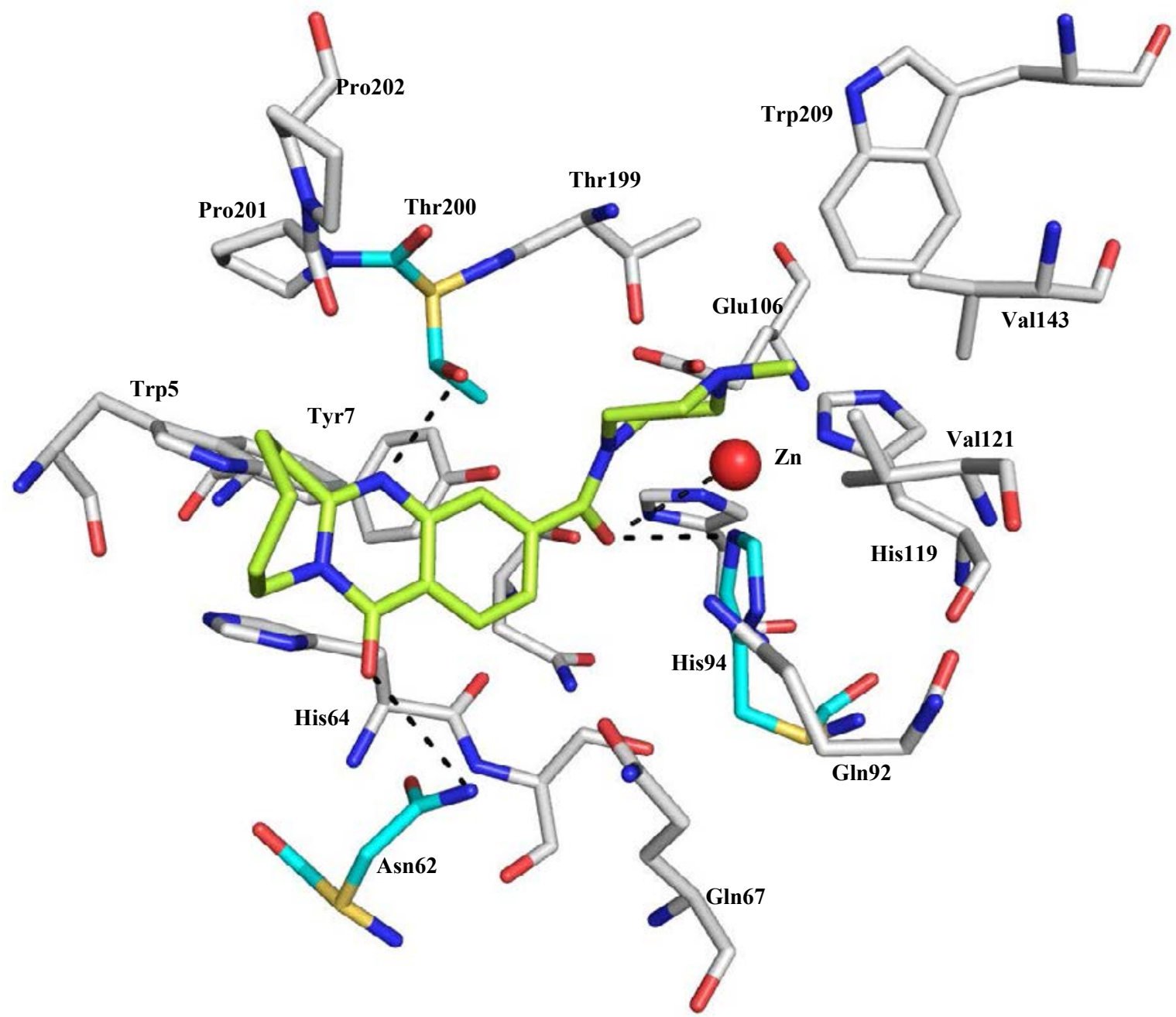

Figure 5: Stereo view of best novel hit (ZINC03363328) docked at CAIX active site. The active site residues Asn-62, Glu-106, His-94, Thr-200 and Zn ${ }^{+2}$ are involved in polar interaction. Val-121, Val-143, Leu-91, Leu-135 and Trp-209 are involved in hydrophobic interaction with methylpiperazine of ZINC03363328. The quinazoline and azepane ring are precisely fitted in upper hydrophobic domain of CAIX active site, composed with Leu-141, Pro-201,202 and Trp-5, Tyr-209 and Phe-245. 
<smiles>CC(=O)Nc1nnc(S(N)(=O)=O)s1</smiles>

Acetazolamide

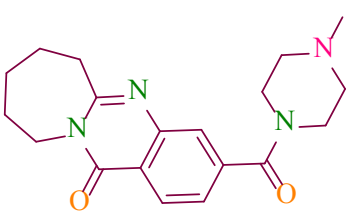

ZINC03363328
Figure 6: Geometric constraints of hydrogen bond donor/acceptor atoms for CAIX inhibitor Acetazolamide and novel hit (ZINC03363328) defined at AutoDock 4.2. The pink atom represents $\mathrm{sp}^{3}$ donor/acceptor atoms while orange and green represent $\mathrm{sp}^{2}$ acceptor and donor atoms, respectively.

Other a azepan derivative hit ZINC12941947, also showed promising pharmacokinetic score. Piperazine ring is generally substituted with compounds to enhance their water solubility. The best docking pose of ZINC03363328, having docking score $(\Delta \mathrm{G}=-11.82 \mathrm{kcal} / \mathrm{mol} ; \mathrm{Ki}=2.32$ $\mathrm{nM}$ ) showed polar interaction with Asn-62, His-94, Thr200 and $\mathrm{Zn}^{+2}$. Methylpiperazine ring is oriented towards lower hydrophobic domain of the active site, surrounded with Leu-91, Leu-93, Leu-198, Val-121 and Trp-209 (Figure 5). Azapane ring enclosed with Trp-5, Leu-141, Val-143, Pro-201, Pro-202 belong to upper hydrophobic domain CAIX active site. The core quinazolin ring having aromatic interaction with Trp-5, Tyr-7 and Phe-245, is also involved in polar interaction with Asn-62, His-94. The geometric constrains for hydrogen bond donor/ acceptor ( $s p^{3}$ donor/acceptor and $s p^{2}$ donor/acceptor) are described in Figure 6. Hence, our results suggest that a conceptual adjustment of these hits may lead to design a novel and potent CAIX inhibitor.

\section{Conclusions}

In this study, 3D pharmacophore hypotheses were generated from active site residues involved in CAIX inhibitors interaction with the protocol implemented in LigandScout 3.1. This method have enough potency to generate pharmacophore model with single docked pose of ligand. However, multiple inhibitors are taken here to capture all possible active site residues of CAIX involve in inhibitor interaction. Docked pose of the best pharmacophore characterized by PI (1), HBD (2), HBA (3), H (2) and AR (1) are essential features for CAIX inhibitor. All selected novel hits against ZINC database are well mapped with n-2 feature with relative hit score $>.95$, showed a good predictive ability of pharmacophore. The observed hits are precisely sorted according to their binding affinity $(\Delta \mathrm{G} \geq 10 \mathrm{kcal} / \mathrm{mol} ; \mathrm{Ki} \geq 10 \mathrm{nM})$ and spatial fitting within the active site. The best ten lead molecules, having energetically favorable interaction with active site, His-94, His-119, Glu-106, Thr199, Thr-200 and $\mathrm{Zn}^{2+}$, are selected as novel hits for CAIX. ADMET analysis was used to check the lead candidates for their drug likeness and bioavailability. Finally, based on the consensus scoring values, critical interactions with the active site residues, and predicted activity values, five compounds (ZINC03363328, ZINC08828920, ZINC12941947, ZINC03622539 and ZINC16650541) are proposed as potent CAIX inhibitors. These results suggest that the application of chemoprint based pharmacophore could assist in selection of potential leads for rational design of CAIX inhibitors in cancer therapy.

\section{Acknowledgments}

AP is thankful to the UGC (BSR grant), Delhi, India, for providing the Dr. DS Kothari Post-doctoral fellowship to carry this work. The authors are thankful to Inte: Ligand support team for providing academic evaluation license of LigandScout 3.1/OMEGA, used effectively for pharmacophore modeling. $\mathrm{MIH}$ is thankful to the Indian Council of Medical Research for financial assistance.

\section{References}

1. Supuran CT (2008) Carbonic anhydrases: novel therapeutic applications for inhibitors and activators. Nat Rev Drug Discov 7: 168-181.

2. Imtaiyaz Hassan M, Shajee B, Waheed A, Ahmad F, Sly WS (2013) Structure function and applications of carbonic anhydrase isozymes. Bioorg Med Chem 21: $1570-1582$.

3. Alterio V, Hilvo M, Di Fiore A, Supuran CT, Pan P, et al. (2009) Crystal structure of the catalytic domain of the tumor-associated human carbonic anhydrase IX. Proc Natl Acad Sci U S A 106: 16233-16238.

4. McDonald PC, Winum JY, Supuran CT, Dedhar S (2012) Recent developments in targeting carbonic anhydrase IX for cancer therapeutics. Oncotarget 3: 8497.

5. Supuran CT (2007) Carbonic anhydrases as drug targets--an overview. Curr Top Med Chem 7: 825-833

6. Alterio V, Di Fiore A, D'Ambrosio K, Supuran CT, De Simone G (2012) Multiple binding modes of inhibitors to carbonic anhydrases: how to design specific drugs targeting 15 different isoforms? Chem Rev 112: 4421-4468.

7. Maresca A, Temperini C, Vu H, Pham NB, Poulsen SA, et al. (2009) Nonzinc mediated inhibition of carbonic anhydrases: coumarins are a new class of suicide inhibitors. J Am Chem Soc 131: 3057-3062.

8. Mader P, Brynda J, Gitto R, Agnello S, Pachl P, et al. (2011) Structural basis for the interaction between carbonic anhydrase and 1,2,3,4-tetrahydroisoquinolin2-ylsulfonamides. J Med Chem 54: 2522-2526.

9. De Simone G, Supuran CT (2010) Carbonic anhydrase IX: Biochemical and crystallographic characterization of a novel antitumor target. Biochim Biophys Acta 1804: 404-409.

10. Leach AR, Gillet VJ, Lewis RA, Taylor R (2010) Three-dimensional pharmacophore methods in drug discovery. J Med Chem 53: 539-558.

11. Schneider G (2010) Virtual screening: an endless staircase? Nat Rev Drug Discov 9: 273-276.

12. Hughes JP, Rees S, Kalindjian SB, Philpott KL (2011) Principles of early drug discovery. Br J Pharmacol 162: 1239-1249.

13. van de Waterbeemd $\mathrm{H}$, Gifford $\mathrm{E}$ (2003) ADMET in silico modelling: towards prediction paradise? Nat Rev Drug Discov 2: 192-204.

14. Zou J, Xie HZ, Yang SY, Chen JJ, Ren JX, et al. (2008) Towards more accurate pharmacophore modeling: Multicomplex-based comprehensive pharmacophore map and most-frequent-feature pharmacophore model of CDK2. J Mol Graph Model 27: 430-438.

15. Güner OF (2002) History and evolution of the pharmacophore concept in computer-aided drug design. Curr Top Med Chem 2: 1321-1332.

16. Klabunde T, Giegerich C, Evers A (2009) Sequence-derived three-dimensional pharmacophore models for G-protein-coupled receptors and their application in virtual screening. J Med Chem 52: 2923-2932.

17. Hassan MI, Kumar V, Somvanshi RK, Dey S, Singh TP, et al. (2007) Structureguided design of peptidic ligand for human prostate specific antigen. J Pept Sci 13: 849-855.

18. Thakur PK, Kumar J, Ray D, Anjum F, Hassan MI (2013) Search of potential inhibitor against New Delhi metallo-beta-lactamase 1 from a series of antibacterial natural compounds. J Nat Sci Biol Med 4: 51-56.

19. Thakur PK, Hassan I (2011) Discovering a potent small molecule inhibitor for gankyrin using de novo drug design approach. Int $\mathrm{J}$ Comput Biol Drug Des 4: 373-386.

20. Hassan MI, Kumar V, Singh TP, Yadav S (2007) Structural model of human PSA: a target for prostate cancer therapy. Chem Biol Drug Des 70: 261-267.

21. Wolber G, Langer T (2005) LigandScout: 3-D pharmacophores derived from protein-bound ligands and their use as virtual screening filters. J Chem Inf Model 45: 160-169.

22. Accelrys Software Inc. (2012) Discovery Studio Modeling Environment Release 3.5. San Diego: Accelrys Software Inc.

23. SciTegic Pipeline Pilot I (2007.) version 6.1.5, Accelrys, San Diego, CA, USA.

24. Brzozowski Z, Sławiński J, Saczewski F, Innocenti A, Supuran CT (2010) Carbonic anhydrase inhibitors: synthesis and inhibition of the human cytosolic 
Citation: Amresh P, Kumar K, Islam A, Hassan I, Ahmad F (2013) Receptor Chemoprint Derived Pharmacophore Model for Development of CAIX Inhibitors. J Carcinog Mutagen S8: 003. doi:10.4172/2157-2518.S8-003

isozymes I and II and transmembrane isozymes IX, XII (cancer-associated) and XIV with 4-substituted 3-pyridinesulfonamides. Eur J Med Chem 45: 2396 2404.

25. Vullo D, Franchi M, Gallori E, Pastorek J, Scozzafava A, et al. (2003) Carbonic anhydrase inhibitors: inhibition of the tumor-associated isozyme IX with aromatic and heterocyclic sulfonamides. Bioorg Med Chem Lett 13: 1005-1009.

26. Smellie A, Teig SL, Towbin P (1995) Poling: Promoting conformationa variation. Journal of Computational Chemistry.

27. Morris GM, Huey R, Lindstrom W, Sanner MF, Belew RK, et al. (2009) AutoDock4 and AutoDockTools4: Automated docking with selective receptor flexibility. J Comput Chem 30: 2785-2791.

28. Fuhrmann J, Rurainski A, Lenhof HP, Neumann D (2010) A new Lamarckian genetic algorithm for flexible ligand-receptor docking. J Comput Chem 31: 1911-1918.

29. Huey R, Morris GM, Olson AJ, Goodsell DS (2007) A semiempirical free energy force field with charge-based desolvation. J Comput Chem 28: 1145-1152.

30. Luthra PM, Prakash A, Barodia SK, Kumari R, Mishra CB, et al. (2009) In silico study of naphtha [1, 2-d] thiazol-2-amine with adenosine A $2 \mathrm{~A}$ receptor and its role in antagonism of haloperidol-induced motor impairments in mice. Neurosci Lett 463: 215-218.

31. Prakash A, Luthra PM (2012) Insilico study of the A(2A)R-D (2)R kinetics and interfacial contact surface for heteromerization. Amino Acids 43: 1451-1464.

32. Brady GP Jr, Stouten PF (2000) Fast prediction and visualization of protein binding pockets with PASS. J Comput Aided Mol Des 14: 383-401.
33. DeLano WL (2003) PyMOL Reference Manual. DeLano Scientific LLC, San Carlos, CA, USA.

34. Wolber G, Langer T (2005) LigandScout: 3-D pharmacophores derived from protein-bound ligands and their use as virtual screening filters. J Chem Inf Model 45: 160-169.

35. Lu C-L, Lu Zhou, Zi-Cheng Li, Xiang Gao, Wei Zhang (2012) Pharmacophore modeling, virtual screening, and molecular docking studies for discovery of novel Carbonic anhydrase IX inhibitors. Med Chem Res 21: 3417-3427.

36. Wolber G, Seidel T, Bendix F, Langer T (2008) Molecule-pharmacophore superpositioning and pattern matching in computational drug design. Drug Discov Today 13: 23-29.

37. de Graaf C, Vermeulen NP, Feenstra KA (2005) Cytochrome p450 in silico: an integrative modeling approach. J Med Chem 48: 2725-2755.

38. Pajouhesh H, Lenz GR (2005) Medicinal chemical properties of successful central nervous system drugs. NeuroRx 2: 541-553.

39. Marzaro G, Guiotto A, Chilin A (2012) Quinazoline derivatives as potential anticancer agents: a patent review (2007-2010). Expert Opin Ther Pat 22: 223252.

40. Breitenlechner CB, Wegge T, Berillon L, Graul K, Marzenell K, et al. (2004) Structure-based optimization of novel azepane derivatives as PKB inhibitors. $J$ Med Chem 47: 1375-1390.
This article was originally published in a special issue, Anticancer Drugs handled by Editor(s). Dr. Philippe Becuwe, Henri Poincaré's University of Nancy, France 